\title{
Enhancement of the catalytic activity of Isopentenyl diphosphate isomerase (IDI) from Saccharomyces cerevisiae through random and site-directed mutagenesis
}

\author{
Hailin Chen ${ }^{1,2}$, Meijie Li ${ }^{1}$, Changqing Liu, Haibo Zhang ${ }^{1 *}$, Mo Xian ${ }^{1 *}$ and Huizhou Liu
}

\begin{abstract}
Background: Lycopene is a terpenoid pigment that has diverse applications in the food and medicine industries. A prospective approach for lycopene production is by metabolic engineering in microbial hosts, such as Escherichia coli. Isopentenyl diphosphate isomerase (IDI, E.C. 5.3.3.2) is one of the rate-limiting enzymes in the lycopene biosynthetic pathway and one major target during metabolic engineering. The properties of IDIs differ depending on the sources, but under physiological conditions, IDls are limited by low enzyme activity, short half-life and weak substrate affinity. Therefore, it is important to prepare an excellent IDI by protein engineering.

Results: Directed evolution strategy (error-prone PCR) was utilized to optimize the activity of Saccharomyces cerevisiae IDI. Using three rounds of error-prone PCR; screening the development of a lycopene-dependent color reaction; and combinatorial site-specific saturation mutagenesis, three activity-enhancing mutations were identified: $\mathrm{L} 141 \mathrm{H}$, Y195F, and W256C. L141H, located near the active pocket inside the tertiary structure of IDI, formed a hydrogen bond with nearby $\beta$-phosphates of isopentenylpyrophosphate (IPP). Phe-195 and Cys-256 were nonpolar amino acids and located near the hydrophobic group of IPP, enlarging the hydrophobic scope, and the active pocket indirectly. Purified IDI was characterized and the result showed that the $K_{m}$ of mutant IDI decreased by $10 \%$ compared with $K_{m}$ of the parent IDI, and $K_{\text {cat }}$ was $28 \%$ fold improved compared to that of the original IDI. Results of a fermentation experiment revealed that mutant IDI had a 1.8-fold increased lycopene production and a 2.1-fold increased yield capacity compared to wild-type IDI.
\end{abstract}

Conclusion: We prepared an engineered variant of IDI with improved catalytic activity by combining random and site directed mutagenesis. The best mutants produced by this approach enhanced catalytic activity while also displaying improved stability in pH, enhanced thermostability and longer half-life. Importantly, the mutant IDI could play an important role in fed-batch fermentation, being an effective and attractive biocatalyst for the production of biochemicals.

Keywords: Isopentenyl diphosphate isomerase, Random mutagenesis, Enzyme activity, Lycopene production, Mevalonate pathway

\footnotetext{
*Correspondence: zhanghb@qibebt.ac.cn; xianmo@qibebt.ac.cn

${ }^{1}$ CAS Key Laboratory of Bio-based Materials, Qingdao Institute of Bioenergy and Bioprocess Technology, Chinese Academy of Sciences, No. 189 Songling Road, Qingdao 266101, People's Republic of China Full list of author information is available at the end of the article
} 


\section{Background}

Lycopene is a widely used carotenoid in the healthcare product market due to its potent antioxidant properties and its links to reduced risk of prostate cancer in humans [1]. Given the recent development of metabolic engineering technologies, one promising approach is the introduction of constituent enzymes of the lycopene biosynthetic pathway into a heterologous host. Cellular lycopene can be synthesized by two different pathways, the mevalonate (MVA) pathway [2] and the 2-methylD-erythritol 4-phosphoric acid (MEP) pathway [3] (Fig. 1). The former is the main synthetic pathway and exists in the cytoplasm, while the latter exists in the chloroplast $[4,5]$. Isopentenyl diphosphate isomerase (IDI,

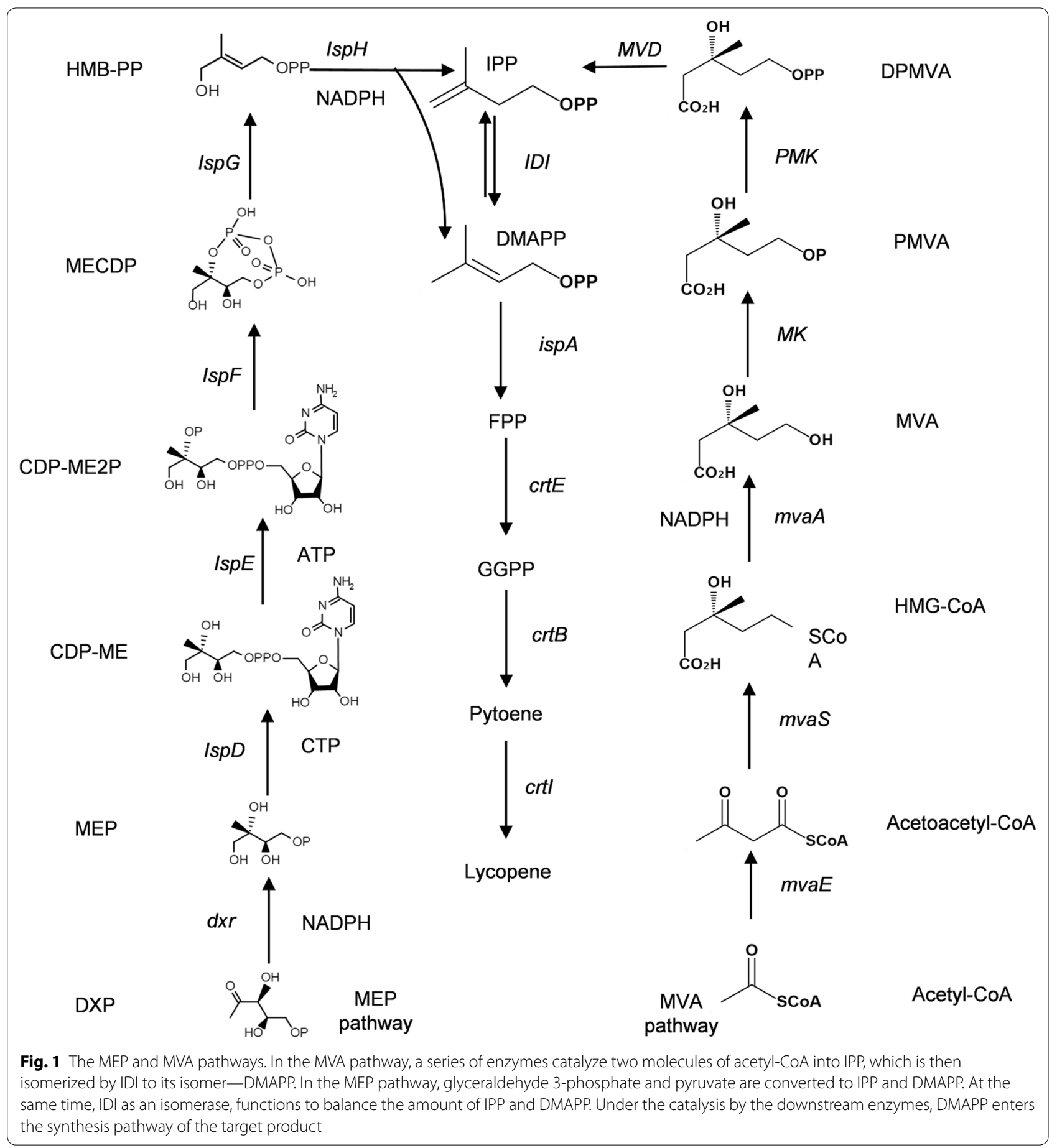


EC 5.3.3.2), which catalyzes the isomerization of isopentenylpyrophosphate (IPP) to dimethylallyl pyrophosphate (DMAPP), is a key rate-limiting enzyme for terpenoid biosynthesis [6]. Depending on different pathways, IDI plays a different but important role in the terpenoid biosynthesis pathway [7]. As reported, the overexpression IDI gene from Escherichia coli and Bacillus licheniformis in E. coli could greatly improve lycopene production [8, 9].

The properties of IDIs differ depending on the sources, but under physiological conditions, IDIs are limited by low enzyme activity, short half-life and weak substrate affinity [10]. Therefore, it is important to prepare an excellent IDI by protein engineering. We chose Saccharomyces cerevisiae IDI (CP008183) as the template, and successfully overexpressed it in E. coli $[11,12]$. In the MVA pathway, 3-hydroxy-3-methyl glutaryl coenzyme A (HMG-CoA) was a key intermediate [13], and the accumulation of HMG-CoA would cause the toxicity to the bottom MVA pathway [14]. In this work, we integrated the bottom portion of the MVA pathway and the lycopene synthesis pathway by constructing a recombinant E. coli strain with the plasmids pET-CHL (containing the bottom MVA pathway) and pAC-LYC (containing key lycopene synthesis genes). An improved high-throughput IDI screening method was established, which not only removed the toxicity of HMG-CoA, but also used the lycopene-dependent color development reaction for selection [15]. Site-directed saturation mutagenesis was conducted to determine the optimal amino acid substitutions at the identified positions [16]. Combinations of site-specific mutations were investigated to identify which combination or combinations of mutations could further enhance IDI enzymatic activity.

\section{Results}

\section{Screening for recombinant strains with high lycopene} production

We performed three cycles of error-prone PCR, and the capacity of the mutation library was always 10,00015,000 strains. Using the lycopene color development reaction as the primary screening method, 20 objective bacteria strains were obtained in each cycle (Fig. 2a).
Eight objective bacteria strains were identified in the first cycle of screening (Fig. 2a), the genomes of which were sequenced and used as a template to perform the second cycle of error-prone PCR. Five objective bacteria strains were identified in the second cycle (Fig. 2a), the genomes of which were sequenced and used as a template to carry out the third cycle of error-prone PCR. Eight objective bacteria strains emerged from the third cycle (Fig. 2a). These 21 strains were sequenced, 17 of which were determined to contain base mutations, and 14 strains contain amino acid mutations. The changes of bases had led to changes in the protein sequence (Additional file 1: Table S2).

\section{Site-directed saturation mutagenesis of residues L141, Y195, and W256}

Substitutions L141, Y195 and W256 were identified as mutations capable of enhancing lycopene production. Separate saturation mutagenesis libraries were constructed using NNK codon degeneracy (20 amino acids/32 codons), which can encode all possible amino acids at positions L141, Y195 and W256 in IDI. Approximately 300 colonies from each of the three libraries were screened to ensure that all possible substitutions were assessed. It has been suggested that screening 94 NNK codon degeneracy mutagenesis colonies will yield a 95\% probability of evaluating all possible 32 outcomes [17].

Five colonies from the V13 library displayed enhanced lycopene production (Fig. 2b), two of which contained histidine substitutions $(\mathrm{L} 141 \mathrm{H})$, while the remaining three contained lysine (L141K) and arginine (L141R), which were the substitutions identified in the consensus library. Amino acid analysis showed that alkaline amino acids were predominate in this screening assay. The $\mathrm{L} 141 \mathrm{H}$ variant displayed 1.05 -fold improvement in lycopene production compared to the wild type, while L141K and L141R showed 0.41-fold and 0.63-fold enhancement. 18 colonies from the Y195 library displayed enhanced lycopene production (Fig. 2b), and all contained substitutions of residues hydrophobic side chains: alanine, leucine, valine, isoleucine, and phenylalanine. Substitutions of Y195-to-Val, -Ile, -Leu or -Ala enhanced lycopene production between 0.22 - and 0.49-fold compare to the wild type, although lycopene production was lower than

\footnotetext{
(See figure on next page.)

Fig. 2 Screening results of three cycles of random mutagenesis (a); saturation mutagenesis at residues L141, Y195, and W256 (b); and critical amino acid substitutions by site-directed mutagenesis (c). a Twenty representative strains are shown for each sequential mutagenesis step. The average relative lycopene production of the mutants increased progressively with every round of mutagenesis. Strains with the highest relative lycopene production in each round were chosen for further analysis. b Twenty representative strains are shown for every position to identify the most advantageous amino acid substitution at these residues. These same colour columns represent different residual activities at the same position. c Single, double and triple mutation enzymes were constructed to determine which were sufficient and necessary for enhanced IDI activity. Every mutant was necessary for IDI and worked well with no interference. Wild-type IDI was used as the control $\left(O D_{475}^{*}\right)$
} 

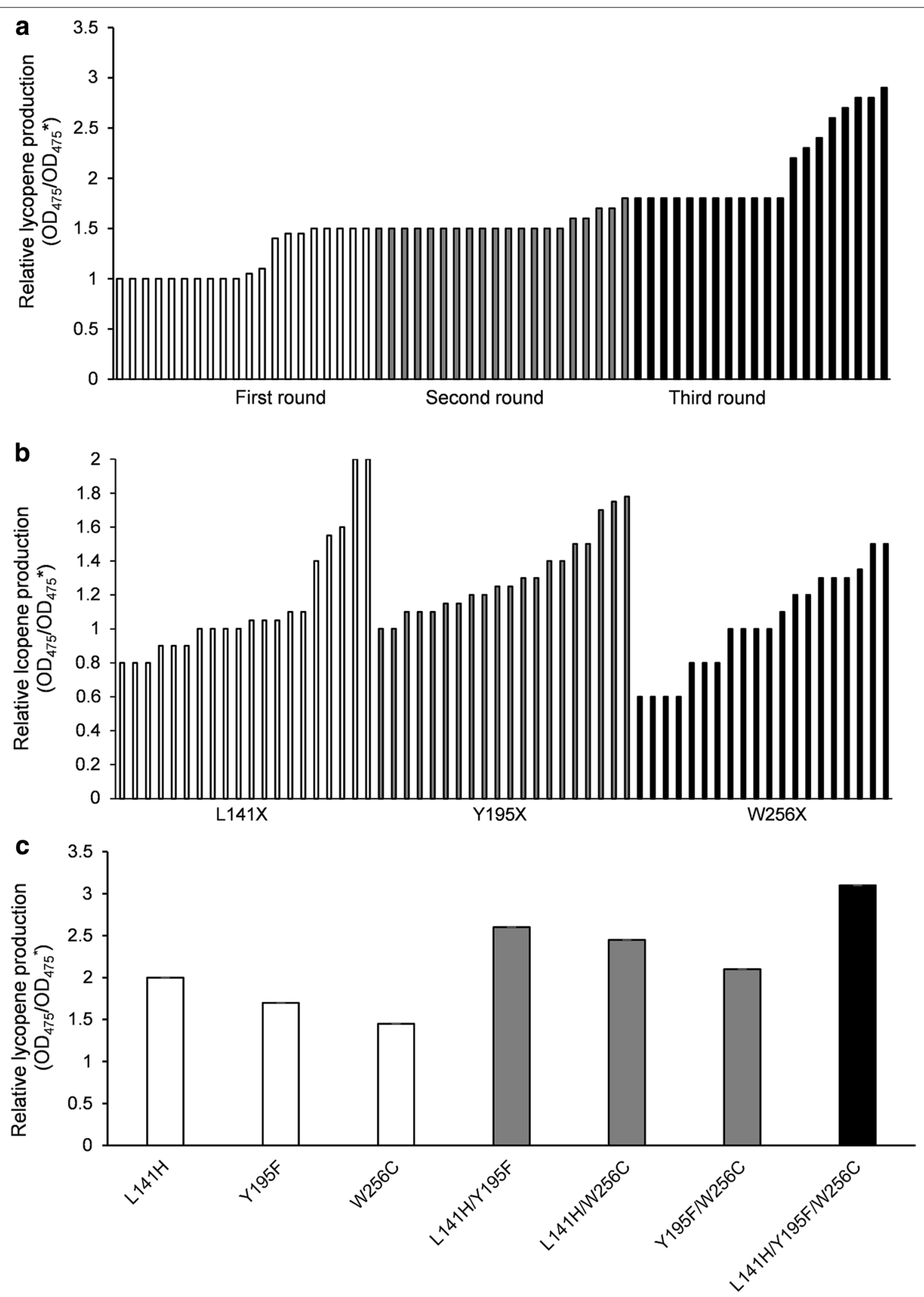
that of Phe148 (which displayed a 0.71-fold increase) as determined in the validation assay. From the W256 screening library, four colonies, including W256C, displayed enhanced lycopene production (Fig. 2b). During the validation screen, variants with improved activity, W256F, W256L and W256I, were found to have a 0.11- to 0.29 -fold increase in lycopene production in comparison with the wild type enzyme. However, W256C showed the highest increase in lycopene production (0.48-fold).

\section{Identification of critical IDI mutations}

The optimal amino acid substitutions for IDI from the saturation mutagenesis screen were L141H, Y195F and W256C. Next, a combinatorial mutagenesis approach was used to identify the IDI variants with the largest increase in lycopene production.

L141H, Y195F and W256C showed 1.10-, 0.71- and 0.47-fold higher lycopene production compared with the wild type, respectively (Fig. 2c), indicating that these individual mutations were all beneficial and improved lycopene production. Moreover, L141H/Y195F, L141H/ W256C and Y195F/W256C showed 2.03-, 1.65- and 1.14fold higher lycopene production, respectively (Fig. 2c), indicating that double mutation combinations could further improve lycopene production. Furthermore, no double mutation combination decreased production. Finally, the triple mutant L141H/Y195F/W256C showed the highest improvement (3.13-fold of wild type) (Fig. 2c). In summary, the enhanced catalytic activity of mutant IDI resulted from additive mutations at residues 141, 195 and 256.

\section{Determination of kinetic parameters}

The kinetic parameters of IDI and triple-mutant IDI (L141H/Y195F/W256C) were summarized in Table 1. The $K_{\mathrm{m}}$ of mutant IDI decreased by $10 \%$ from $41.5 \pm 0.39$ to $37.6 \pm 0.17 \mu \mathrm{M}$ [18]. Such a decrease indicated that the affinity of triple mutant IDI for substrate had increased, and the specific activity of the enzyme had risen. The $K_{\text {cat }}$ value increased from $8.27 \pm 0.06$ to $10.57 \pm 0.13 \mathrm{~s}^{-1}$. The catalytic efficiency of the enzyme was therefore enhanced from 0.20 to $0.28 \mathrm{~s}^{-1} \mu \mathrm{M}^{-1}$.

Table 1 Kinetic parameters of IDI and IDI (L141H/Y195F/ W256C)

\begin{tabular}{lcrlc}
\hline Enzyme & $\boldsymbol{K}_{\mathbf{m}}(\boldsymbol{\mu \mathrm { M } )}$ & $\boldsymbol{K}_{\text {cat }}\left(\mathbf{s}^{-\mathbf{1}}\right)$ & $\begin{array}{l}\boldsymbol{K}_{\text {cat }} / \boldsymbol{K}_{\mathbf{m}} \\
\mathbf{s}^{-\mathbf{1}} \\
\left.\boldsymbol{\mu M}^{-\mathbf{1}}\right)\end{array}$ & $\begin{array}{l}\text { Maximum } \\
\text { enzyme activity } \\
(\mathbf{U} / \mathbf{m g})\end{array}$ \\
\hline IDI & $41.5 \pm 0.39$ & $8.27 \pm 0.06$ & 0.20 & $63.87 \pm 1.09$ \\
IDI (L141H/ & $37.6 \pm 0.17$ & $10.57 \pm 0.13$ & 0.28 & $161.60 \pm 2.74$ \\
Y195F/ & & & & \\
W256C) & & & & \\
\hline
\end{tabular}

Triple-mutant IDI (L141H/Y195F/W256C) resulted in 1.40-fold improvement in the $K_{\mathrm{cat}} / K_{\mathrm{m}}$ value. Specific activity of IDI (L141H/Y195F/W256C) was 2.53-fold higher than that of IDI (Table 1). In addition, the expression levels of the wild type and mutated IDI were verified in our study. From Additional file 1: Figure S2, the wild type and mutated IDI had same expression levels. It proved that three mutations didn't affect expression level of IDI, and the expression levels of IDI and IDI (L141H/ Y195F/W256C) didn't affect lycopene production.

\section{Enzymatic properties of wild-type and triple-mutant IDI}

As shown in Fig. 3a, IDI (L141H/Y195F/W256C) had higher activity than WT between $\mathrm{pH} 5.0$ and $\mathrm{pH} 10.0$. The activities of both enzymes increased when $\mathrm{pH}$ was $<7.5$, and decreased when $\mathrm{pH}$ was $>7.5$, suggesting that the optimum $\mathrm{pH}$ for both IDIs was 7.5 (Fig. 3a). The mutations did not affect the optimal pH for IDI activity, but affect IDI activity for the optimal pH. Figure 3b showed that in the $\mathrm{pH}$ range of 5.0-7.5, activity of IDI (L141H/Y195F/W256C) increased rapidly, and while the $\mathrm{pH}$ was $>7.5$, activity decreased. The general trends suggested that IDI (L141H/Y195F/W256C) had better $\mathrm{pH}$ stability propertied compared to wild-type IDI.

As shown in Fig. 4a, the enzymatic activities of wildtype and triple-mutant IDI increased from 25 to $35^{\circ} \mathrm{C}$, reaching a maximum at $35{ }^{\circ} \mathrm{C}$. The activity of both IDIs were reduced at temperature above $35^{\circ} \mathrm{C}$, indicating that the optimal temperature for both IDI variants was $35^{\circ} \mathrm{C}$, and that mutations did not affect the optimal temperature for IDI activity. Interestingly, the activity of IDI (L141H/ Y195F/W256C) was more than 100\% compared to wildtype IDI at the optimal temperature. The thermal stabilities of both IDIs continuously decreased at temperature above $35{ }^{\circ} \mathrm{C}$, while the thermal stability of wild-type IDI decreased more significantly than IDI (L141H/Y195F/ W256C) (Fig. 4b). A possible explanation for this finding may be that a salt bridge was formed by the mutations, which enhanced the rigidity of the enzyme (Fig. 7b).

Mutants exhibited improved thermostability (Fig. 4b). Therefore, the thermostability of IDI (L141H/Y195F/ W256C) was further analyzed by measuring the half-lives $\left(\mathrm{T}_{1 / 2}\right)$ at $30-50{ }^{\circ} \mathrm{C}$. Table 2 listed the $\mathrm{T}_{1 / 2}$ values of wildtype IDI and IDI (L141H/Y195F/W256C) at different temperatures. $\mathrm{T}_{1 / 2}$ at $25-50{ }^{\circ} \mathrm{C}$ were $2.6-6$ times longer than the WT. Enhancement of the thermostability of IDI is particularly interesting, as it will extend the shelf life of IDI in the fermentation process.

\section{Lycopene production of mutant and wild-type IDI in batch fermentation assays}

The performance of IDI (L141H/Y195F/W256C) was ultimately evaluated in terms of the final lycopene yield 

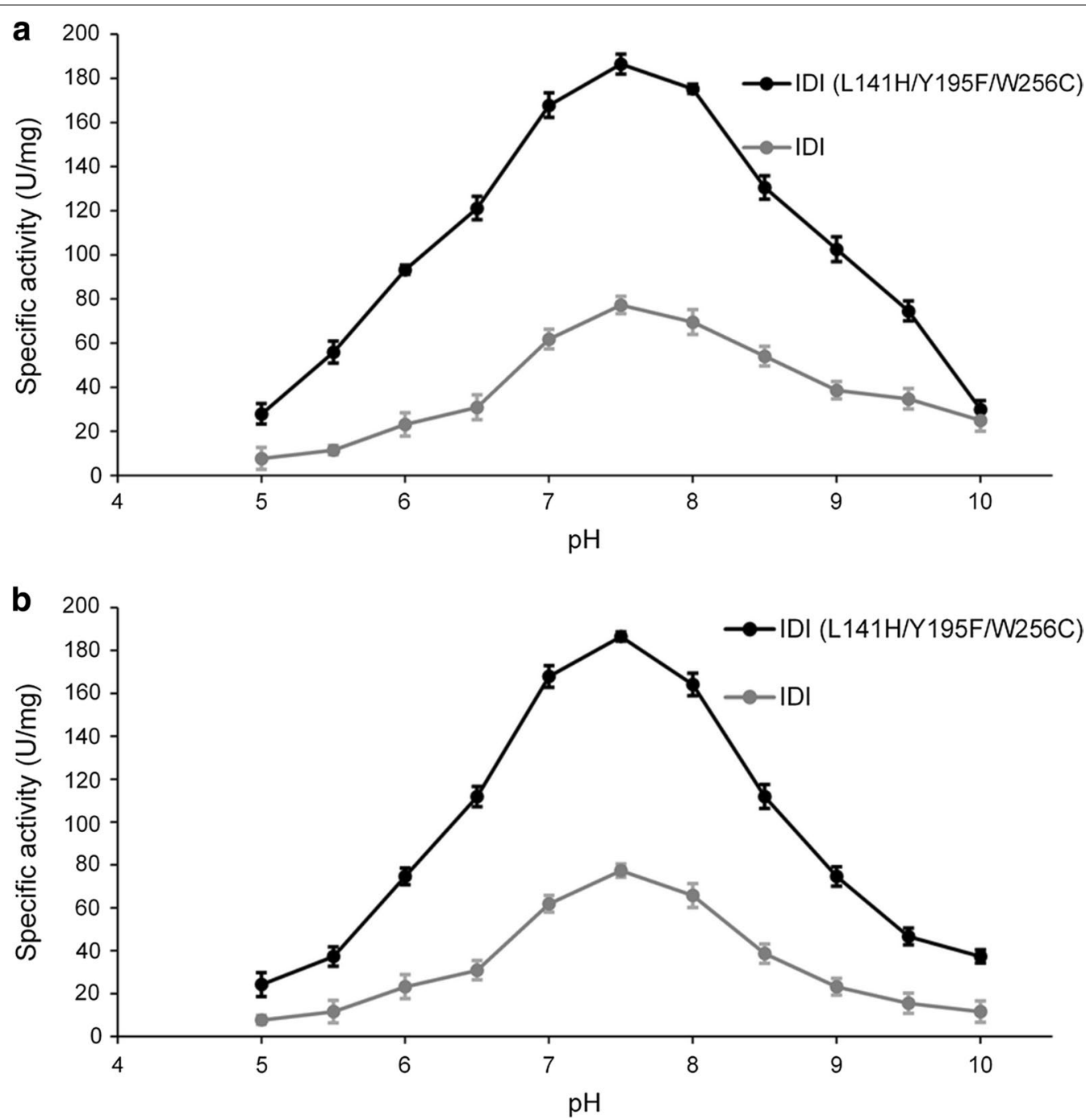

Fig. 3 Effect of pH on the activity (a) and stability (b) of IDI (L141H/Y195F/W256C) and IDI. a The optimum pH was 7.5 measured at $25^{\circ} \mathrm{C}$. The mutations did not affect the optimal pH for IDI activity, but affect IDI activity for the optimal pH. b The general trends suggested that IDI (L141H/ Y195F/W256C) had better pH stability propertied compared to wild-type IDI

and production through fed fermentation. For these experiments, the recombinant strain containing wildtype IDI was named CHL-1, and the recombinant strain containing IDI (L141H/Y195F/W256C) was named CHL-2. Fed-batch fermentations were carried out to test the suitability and stability of IDI (L141H/Y195F/ W256C) for the improved production of lycopene.

As shown in Fig. 5a, during the fed-batch stage, the biomass showed a steady increase, while the lycopene accumulation was significantly increased. After $90 \mathrm{~h}$ of fermentation, the strain CHL-2 had 1.8-fold-improved lycopene production above the original strain CHL-1 (Fig. 5a), while the lycopene yield of CHL-2 was 2.1fold higher than that of CHL-1 (Fig. 5b). Although the fermentation process was stopped after $90 \mathrm{~h}$, the lycopene accumulation in CHL-1 lasted for $70 \mathrm{~h}$, while lycopene accumulation in CHL-2 was continuous throughout the whole fermentation process (Fig. 5a). The lycopene production and lycopene accumulation time suggested that the engineered triple-mutant IDI efficiently improved lycopene production.

\section{Structural simulation and molecular docking of IDI}

In order to provide insight into the molecular basis for increased enzymatic activity of IDI (L141H/Y195F/ W256C), in silico structural modelling and molecular docking were performed by using the $3 \mathrm{D}$ structure of IPP isomerase from E. coli (PDB ID: 2ICJ), as a model 

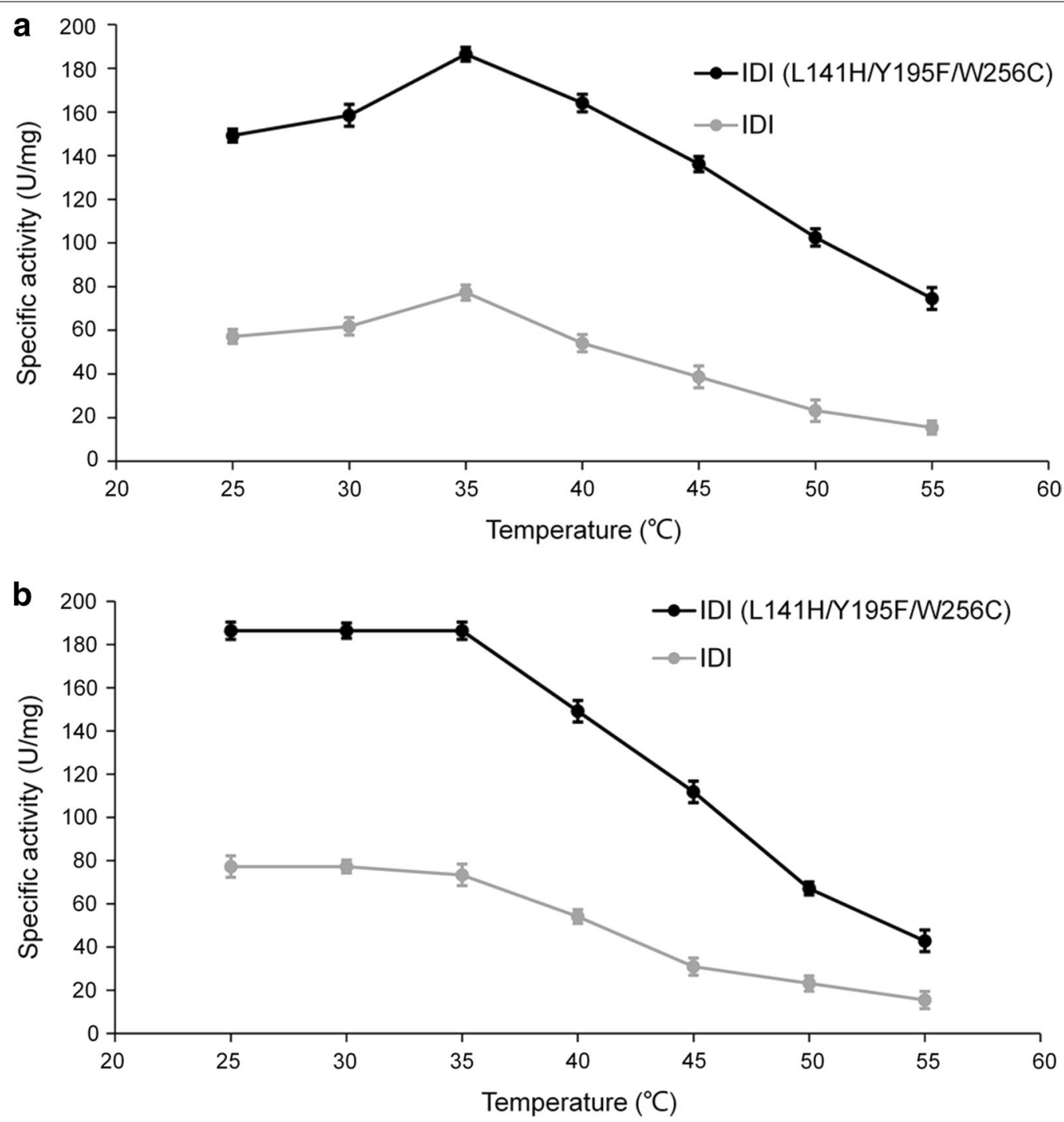

Fig. 4 Effect of temperature on the activity (a) and stability (b) of IDI (L141H/Y195F/W256C) and IDI. a The optimal temperature was $35^{\circ} \mathrm{C}$, and that mutations did not affect the optimal temperature for IDI activity. $\mathbf{b}$ The thermal stabilities of both IDIs continuously decreased at temperature above $35^{\circ} \mathrm{C}$, while the thermal stability of wild-type IDI decreased more significantly than IDI (L141H/Y195F/W256C)

Table 2 The half-lives ( $\left.T_{1 / 2}\right)$ of IDI and IDI (L141H/Y195F/ $\mathrm{W} 256 \mathrm{C}$ ) at different temperatures

\begin{tabular}{lrll}
\hline $\begin{array}{l}\text { Temperature } \\
\left({ }^{\circ} \mathbf{C}\right)\end{array}$ & IDI & $\begin{array}{l}\text { IDI (L141H/ } \\
\text { Y195F/W256C) }\end{array}$ & $\begin{array}{l}\text { Fold } \\
\text { improvement }\end{array}$ \\
\hline 30 & $32.7 \mathrm{~h}(48.9 \%)$ & $85.0 \mathrm{~h}(50.1 \%)$ & 2.6 \\
35 & $15.3 \mathrm{~h}(51.0 \%)$ & $45.6 \mathrm{~h}(50.7 \%)$ & 3.0 \\
40 & $4.0 \mathrm{~h}(50.3 \%)$ & $32.9 \mathrm{~h}(49.8 \%)$ & 4.7 \\
45 & $61 \mathrm{~min}(50.8 \%)$ & $14.1 \mathrm{~h}(51.0 \%)$ & 13.8 \\
50 & $10 \mathrm{~min}(49.7 \%)$ & $2.67 \mathrm{~h} \mathrm{(49.5 \% )}$ & 16.0 \\
\hline
\end{tabular}

template of IDI. The results of in silico structural modelling was shown in Fig. 6a. His-93, His-104, Cys-139, Glu-205, Glu-207, and Mg-302, Mg-400 formed an active pocket of IDI, identified to be the active sites (Fig. 6b). Cys-139 and Glu-207 were related to the protonation and deprotonation of substrate IPP, which were the most important sites of IDI [19]. In addition, the residual groups of Lys-89, Arg-123 and Lys127 were hydrogenbonded to both $\alpha$ - and $\beta$-phosphates of IPP (Fig. 7a). Hydrophobic interaction of Ala-106, Phe-107 and Cys139 with IPP could not only increase the binding capacity 

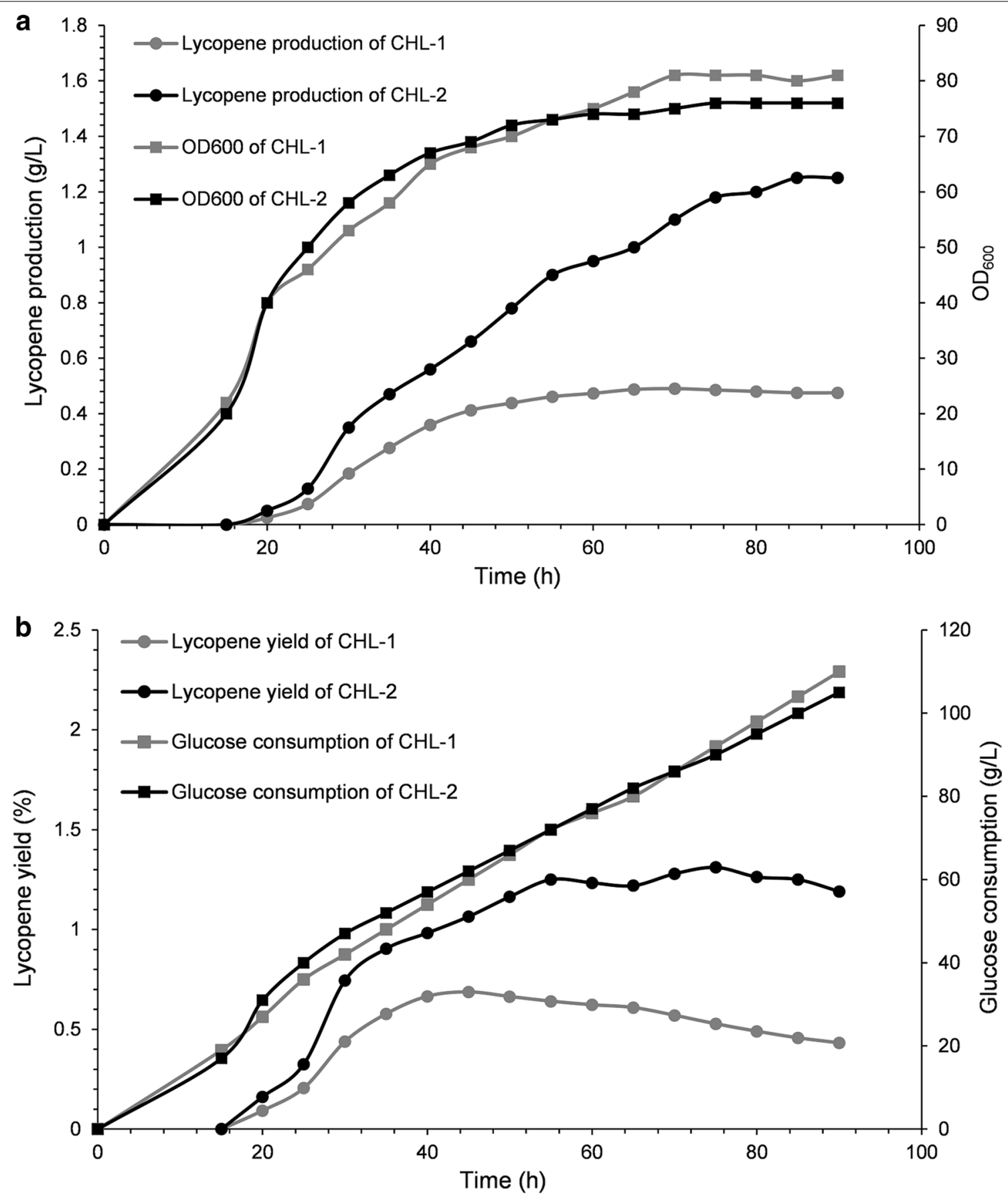

Fig. 5 Cell growth rates, lycopene productions and lycopene yields, substrate consumption rates during aerobic fed-batch fermentation. a The cell growth rates of $\mathrm{CHL}-1$ and $\mathrm{CHL}-2$ were similar, and lycopene production of $\mathrm{CHL}-2$ were approximately 2.8 -fold of $\mathrm{CHL}-1$. $\mathbf{b}$ The substrate rates of $\mathrm{CHL}-1$ and $\mathrm{CHL}-2$ were similar, while final lycopene yield of $\mathrm{CHL}-2$ was approximately 3.1 -fold of $\mathrm{CHL}-1$

of enzyme and substrate, but also promote the correct refolding of protein (Fig. 7c). The residues at positions 141, 195 and 256, which are near the catalytic center of IDI in its 3D structure, would directly interact with the active center.
His-141 was located near the active pocket inside the tertiary structure of IDI (Fig. 6c). It was an alkaline amino acid, therefore it could bind the $\beta$-phosphate of IPP (negatively charged) (Fig. 7b). Binding of IPP was strengthened through the formation of additional hydrogen bond. 


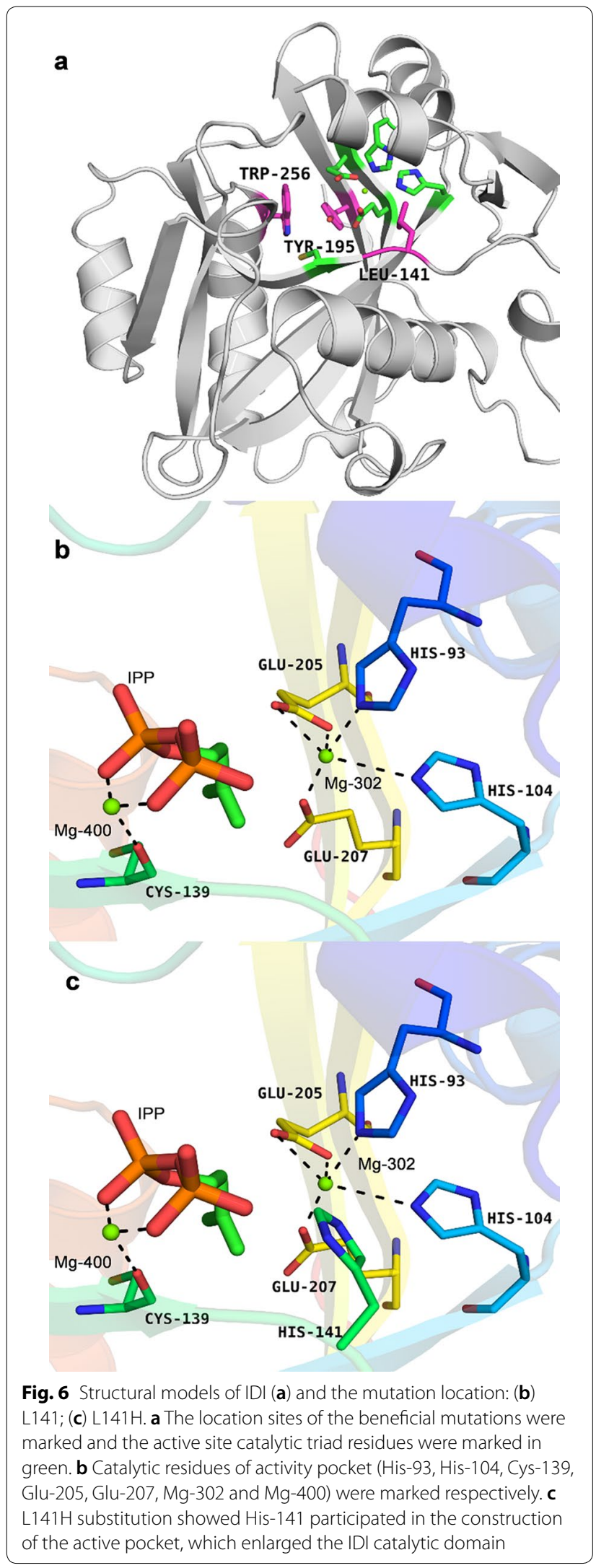

Because of the hydrogen bond of His-141 and IPP, His141 became one of the active sites (Fig. 6c) [20]. His-141 was near Mg-302 of the active pocket, and Mg-302 and His-141 had like electric charges. Thus Mg-302 repelled His-141, and it could be inferred that the hatchway for substrate molecules to enter the enzyme active center became larger and the hindrance reduced accordingly, thus enhancing the enzyme activity. The molecular docking results revealed that the binding energy between the substrate and the mutant enzyme was increased to $8.87 \mathrm{kcal} / \mathrm{mol}$ from the original $6.87 \mathrm{kcal} / \mathrm{mol}$ [21]. The increase in the binding energy could likely explain the improved substrate affinity.

Phe-195 and Cys-256 were nonpolar amino acids and located near the hydrophobic group of IPP (Fig. 7d). Due to hydrophobicity of Phe-195 and Cys-256, Ala-106, Phe107 and Cys-139 could fixed substrate better [22]. The amino acid substitutions at the position of 195 and 256 enlarged the hydrophobic scope, and the active pocket indirectly therefore enhancing enzymatic activity to some extent.

\section{Discussion}

The beneficial substitutions at positions 141, 195 and 256 were found by error-prone PCR (Fig. 2a) and the optimal amino acid substitutions at these positions were further analyzed to investigate their interactions and the molecular mechanisms behind this improved lycopene production (Fig. 2b). As shown in Fig. 2c, IDI worked better with any combination of single, double or triple mutations at these residues. This suggested that $\mathrm{L} 141 \mathrm{H}, \mathrm{Y} 195 \mathrm{~F}$ and W256C were distinct mutations that contribute considerably to the enhanced activity of mutant IDI.

Enzymatic properties showed that the three optimal substitutions, L141H, Y195F and W256C, were beneficial for the improved activity and thermostability of IDI. 2.53 -fold improvement in catalytic activity suggested that three mutations enhanced performance of mutant IDI in the biocatalysis of substrates. Although the mutations did not affect the optimal $\mathrm{pH}$ and temperature for IDI, but affect IDI activity for the optimal $\mathrm{pH}$ and temperature significantly. This suggested that enhanced enzymatic activity was primarily a result of two main factors: (1) improved affinity between the substrate and enzyme caused by mutations (L141H, Y195F and W256C) and (2) increased enzyme stability due to mutations within the hydrogen bond and hydrophobic interaction [23]. In addition, the $\mathrm{T}_{1 / 2}$ values at $35^{\circ} \mathrm{C}$ of IDI and IDI (L141H/ Y195F/W256C) expressed in E. coli were 15.3 and $45.6 \mathrm{~h}$, respectively, indicating that the hydrogen bond and hydrophobic interaction of L141H, Y195F, and W256C could improve its thermostability. 


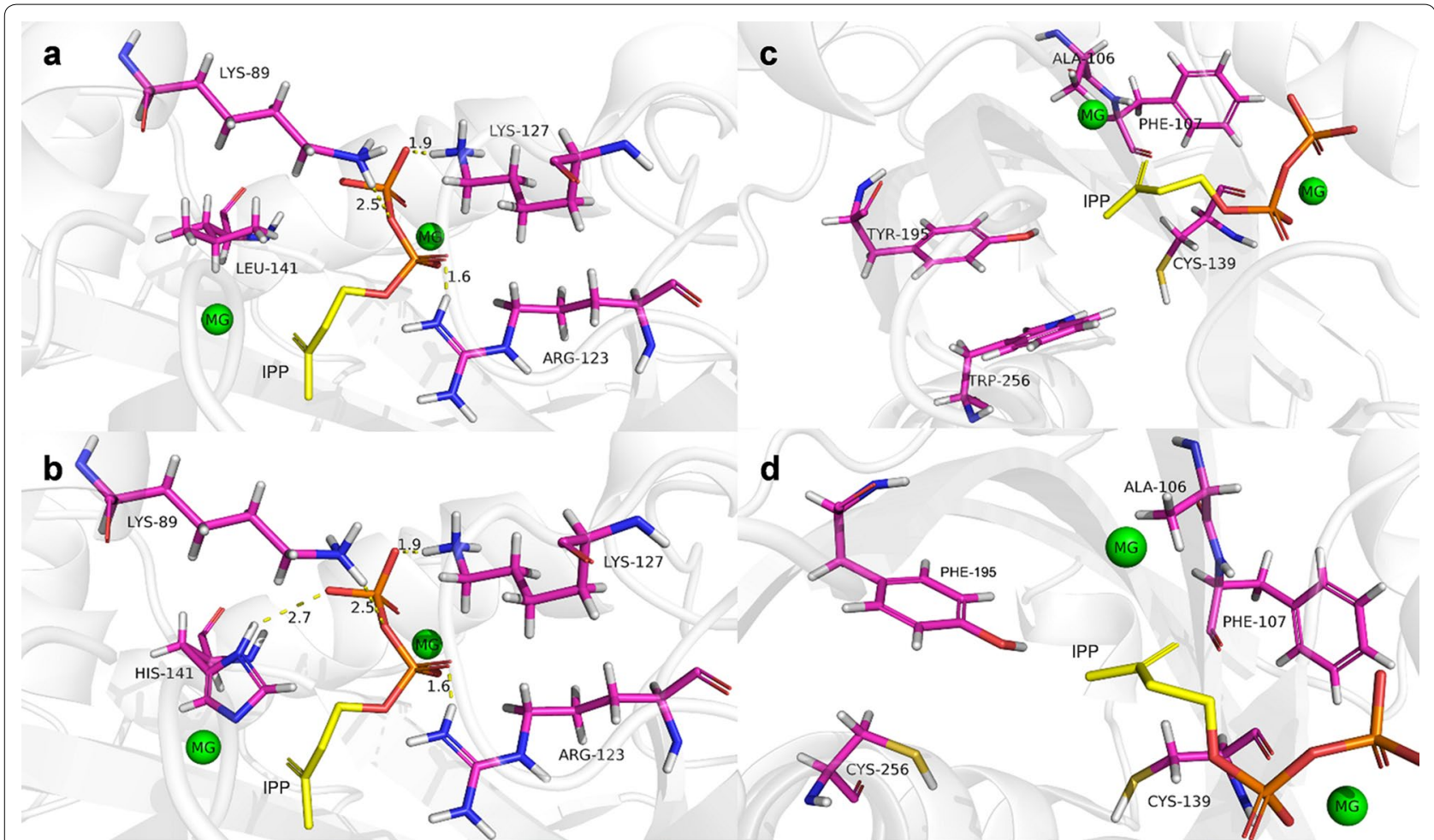

Fig. 7 Structural models of mutation locations: a L141; b L141H; c Y195, W256; d Y195F, W256C. a, b L141H substitution showed His-141 formed a hydrogen bond with $\beta$-phosphates of IPP, strengthening binding of IPP. c, d Phe-195 and Cys-256 substitution was located near the hydrophobic group of IPP and improved the stability of binding of IPP

The fermentation results revealed enhanced performance of mutant IDI with regards to the production and final yield of target product. This was the ultimate goal of directed evolution of IDI. As shown in Fig. 5a, the strain CHL-2 had improved lycopene production compared to the original strain CHL-1, indicating that triple-mutant (L141H/Y195F/W256C) could translate improved enzymatic activity into the improvement of lycopene production successfully in an expanded system. For industrial application, this was a considerable advantage. Under these conditions, finally the lycopene yield of CHL-2 was higher than that of CHL-1 (Fig. 5b) [24]. This suggested that more glucose would be used to synthesize lycopene, another very important consideration for industrial application. This promised more substrate would be translated into target product rather than by-product, thus minimizing waste.

A 3D homology model of IDI was created to improve our understanding of the mutagenesis effect on IDI activity (Fig. 6a). According to the model, all mutations, $\mathrm{L} 141 \mathrm{H}, \mathrm{Y} 195 \mathrm{~F}$ and W256C are located near the activity pocket and may have interactions with IPP. Generally, three mutations have a cumulative effect for IDI (Fig. 2c). In this work, the respective change at position 141 could improve the structural stability through hydrogen bond (Fig. 7a, b), and increasing binding energy would be used to reduce the activation energy, to increase the substrate affinity, and the $K_{\mathrm{m}}$ value of IDI (L141H/Y195F/W256C) decreased (Table 1). The residues 195 and 256 with their surroundings (Ala-106, Phe-107 and Cys-139) (Fig. 7c) determine the conformation of IDI. The hydrophobic interactions of Phe195 and Cys256 with their surroundings had a better effect on the local structural stability (Fig. 7d) in comparison with those of Tyr195 and Trp256 (Fig. 7c). Therefore, Y195F/W256C may improve the thermostability of IDI.

\section{Conclusion}

In conclusion, we have successfully engineered IDI to improve its catalytic activity and lycopene production via a random mutagenesis approach. In this study, we used error-prone PCR to obtain a mutant IDI library and developed a lycopene-dependent color development reaction to screen for activity-enhancing mutations. The best mutants produced by this approach maintained catalytic activity while also displaying improved thermoand $\mathrm{pH}$ stability and longer half-life. These properties could make it a potent and attractive biocatalyst for the 
production of biochemicals. Fermentation experiments revealed that the lycopene production of triple-mutant IDI was 1.8-fold higher than wild-type IDI; however, due to the inherent deficiency of error-prone PCR, it was difficult to substantially increase IDI activity. The IDI performance in lycopene production could be further optimized by using additional or alternative protein engineering techniques, such as DNA shuffling [25], rational design [26], B-FIT [27], or ISM [28] approaches. The X-ray crystal structures of these engineered enzymes will also be further investigated to further elucidate the molecular mechanisms that govern catalytic activity.

\section{Methods}

\section{Chemicals and materials}

Restriction enzymes, T4 DNA ligase, Taq DNA polymerase, and PCR reagents were purchased from Takara Biomedical Technology (Beijing) Co., Ltd., and primers were synthesized by Jin Weizhi Biological Technology Co., Ltd. Gel Extraction Kit, PCR product DNA Purification Kit and Plasmid Mini Kit were from Omega Bio-Tek. All reagents and chemicals were of analytical grade and, unless otherwise stated, obtained from commercial sources.

\section{Bacterial strains and plasmids}

Details of the plasmids and bacterial strains used in this study were shown in Additional file 1: Table S1. The E. coli strains BL21(DE3) and DH5 $\alpha$ (Invitrogen) were used for plasmid preparation, protein overexpression/lycopene fermentation, respectively. Gene cloning was performed using S. cerevisiae (Invitrogen). Recombinant plasmid pCLpTrcUpper [29] was built and stored by our laboratory. Recombinant plasmid PAC-LYC [30] was provided by University of Maryland. Vector pETDeu-1 was purchased from Invitrogen.

\section{Plasmid construction}

The four key enzymes that represent the bottom portion of the MVA pathway were combined for the lycopene color development reaction screening. Through the bottom pathway, the primary phosphorylation by mevalonate kinase (MK, EC2.7.1.366) and secondary phosphorylation by phospho-mevalonate kinase (PMK, EC2.7.4.2) converts MVA to diphosphomevalonate (DPMVA). Isomerization by isopentenyl diphosphate isomerase (IDI, EC5.3.3.2) and decarboxylation by diphospho-mevalonate decarboxylase (MVD, EC4.1.1.33) produces dimethylallyl pyrophosphate (DMAPP).

Four S. cerevisiae genes (PMK, MVD, MK, and IDI; ATCC201508D) were cloned into the pETDeut-1 vector. The pET-CHL1 plasmid was constructed by inserting PMK (ERG8) into the Bgl II and Aat II sites of pETDuet-1 with ERG8_F and ERG8_R primers. The plasmid
pET-CHL2 was created by inserting MVD (ERG19) into the Aat II and Xho I sites of pET-CHL1 with primers ERG19_F and ERG19_R. The pET-CHL3 plasmid was established by inserting MK (ERG12) into the Sac I and Not I sites of the pET-CHL2 vector with primers ERG12_F and ERG12_R. The pET-CHL plasmid was built by the PCR amplification IDI (IDI) using primers IDI_F and IDI_R and cloning it into the $S a c$ I and $S a l$ I sites of the plasmid pET-CHL3.

\section{Construction of the mutation library}

Error-prone PCR was used to introduce random mutations into IDI within the pET-CHL vector. The errorprone PCR reaction was as follows: $10 \times$ error-prone PCR buffer; $0.5 \mathrm{mmol} / \mathrm{L}$ dATP and dGTP; $2.5 \mathrm{mmol} / \mathrm{L} \mathrm{dCTP}$ and dTTP; 40 pmol of each primer (IDI_F and IDI_R); $3 \mathrm{mmol} / \mathrm{L} \mathrm{MgCl}_{2} ; 0.2 \mathrm{mmol} / \mathrm{L} \mathrm{MnCl}_{2} ; 2.5 \mathrm{U}$ Taq DNA polymerase, and water. The hot-start PCR amplification program annealed primers at $62{ }^{\circ} \mathrm{C}$ for $2 \mathrm{~min}$, and product extension was performed at $72{ }^{\circ} \mathrm{C}$ for $2 \mathrm{~min}$; this cycle was repeated 35 times.

After error-prone PCR amplification, PCR products and the pET-CHL vector were purified by $\mathrm{Sac}$ I and $\mathrm{Sal}$ I digestion, ligated, and transformed into competent DH5 $\alpha$ E. coli, and positive colonies were selected and amplified to purify mutant plasmids.

\section{Screening of mutants with high lycopene production}

The pET-CHL and pAC-LYC plasmids were transformed in $E$. coli BL21(DE3), and the transformation reaction was spread onto LB-agar plates, which were cultured for $15 \mathrm{~h}$ at $37^{\circ} \mathrm{C}$ to generate the mutant screening library. Colonies were considered candidate strains and were cultured in 96-well plates in $300 \mu \mathrm{L}$ of LB medium supplemented with MVA ( $3 \mathrm{~g} / \mathrm{L})$, ampicillin $(100 \mu \mathrm{g} / \mathrm{mL})$, and chloramphenicol $(50 \mu \mathrm{g} / \mathrm{mL})$ before incubation for $8 \mathrm{~h}$ at $37^{\circ} \mathrm{C}$. Protein expression was then induced with $0.05 \mathrm{mmol} / \mathrm{L}$ Isopropyl $\beta$-D-1-thiogalactopyranoside (IPTG) for $48 \mathrm{~h}$, and then the cells were pelleted by centrifugation at $3000 \times g$ for $5 \mathrm{~min}$. Next, the pellet was resuspended in $200 \mu \mathrm{L}$ of acetone, and the mixture was incubated for $15 \mathrm{~min}$ at $55{ }^{\circ} \mathrm{C}$, and then centrifuged at $10,000 \times g$ for $3 \mathrm{~min}$. Finally, $200 \mu \mathrm{L}$ of the supernatant was measured at $\mathrm{OD}_{475}$ on a spectrophotometer to indirectly determine the relative lycopene content [31]. Supernatant isolated from the control strain did not absorb light at $475 \mathrm{~nm}$ under the same conditions.

\section{Site-specific saturation mutagenesis}

To construct IDI libraries containing all possible amino acid substitutions at residues L141, Y195, and W256 of IDI, the target codon was replaced with an NNK degenerate codon (K represents $\mathrm{G}$ or $\mathrm{T}$ and $\mathrm{N}$ represents $\mathrm{A}, \mathrm{T}$, 
G, or C) using the QuikChange II site-directed mutagenesis kit. The IDI-L141, IDI-Y195, and IDI-W256 libraries were produced with the L141-F/R, Y195-F/R, and W256$\mathrm{F} / \mathrm{R}$ primers, respectively (Additional file 1 : Table S1). The constructs from all three libraries were then transformed into competent BL21(DE3). The original pET-CHL plasmid containing wild-type $I D I$ was used as the template.

\section{Site-directed mutagenesis}

The double mutations L141H/Y195F, L141H/W256C, Y195F/W256C and triple mutation L141H/Y195F/ W256C were created by using the QuikChange II sitedirected mutagenesis kit. The Y195F substitution was introduced into pET-CHL/IDI-L141H using Y195F-F and Y195F-R primers (see Additional file 1: Table S1). E. coli BL21(DE3) competent cells were transformed with the pET-CHL/IDI-L141H/Y195F plasmid using electroporation.

\section{IDI activity assay}

The quantity of isoprene was measured by coupling the acidification and heating of DMAPP with the reactions catalyzed from IPP to DMAPP by IDI. This coupled reaction was monitored subsequently by gas chromatography (GC). For this, 2-mL sealed vials were used and added with $88 \mu \mathrm{L}$ of protein extract, $2 \mu \mathrm{L}$ of $1 \mathrm{M} \mathrm{MgCl}_{2}$, and $10 \mu \mathrm{L}$ of $2.5 \mathrm{mM}$ IPP in an assay buffer (50 mM MOPS, $20 \mathrm{mM} \mathrm{MgCl} 2,5 \%$ glycerol (v/v), pH 7.5). The vials were incubated at $30{ }^{\circ} \mathrm{C}$ for $90 \mathrm{~min}$. The reaction mixtures were acidified by adding $10 \mu \mathrm{L}$ of $85 \% \mathrm{H}_{3} \mathrm{PO}_{4}$ and heated to $70{ }^{\circ} \mathrm{C}$ for $90 \mathrm{~min}$. The resulting isoprene released from DMAPP was measured by GC [32].

\section{Determination of kinetic parameters and effect of temperature and $\mathrm{pH}$ on enzyme activity}

Purified IDI was used to investigate the $V_{\max }, K_{\mathrm{m}}, K_{\text {cat }}$, $\mathrm{pH}$ and thermal stability of the enzyme. The $K_{\mathrm{m}}$ value was determined by double-reciprocal plot method where the concentration range of the substrate was 1-1000 mmol/L [33]. Phosphate buffer $(0.05 \mathrm{~mol} / \mathrm{L}$, $\mathrm{pH}$ 5.0-8.0), $0.05 \mathrm{~mol} / \mathrm{L}$ Tris- $\mathrm{HCl}$ buffer $(\mathrm{pH} 8.0-9.0)$ and $0.05 \mathrm{~mol} / \mathrm{L}$ carbonate buffer $(\mathrm{pH} 9.0-10.0)$ were prepared to determine the optimum $\mathrm{pH}$ required for IDI activity [34]. When determining the stability of IDI with altered $\mathrm{pH}$ conditions, $0.25 \mathrm{U}$ enzyme solution was respectively added to the above buffer solutions with different $\mathrm{pH}$ values, and then the solutions were stored at $25{ }^{\circ} \mathrm{C}$ for $1 \mathrm{~h}$ to determine activity of the enzyme under standard conditions. When determining thermal stability, $0.25 \mathrm{U}$ enzyme solution underwent heat preservation at $25-55^{\circ} \mathrm{C}$ for $1 \mathrm{~h}$ to determine the IDI activity under standard conditions. In addition, the $\mathrm{T}_{1 / 2}$ of IDI and mutant IDI at $30-50^{\circ} \mathrm{C}$ were measured. The systems containing $0.1 \mathrm{mg} / \mathrm{mL}$ purified IDI $(0.05 \mathrm{~mol} / \mathrm{L}$ phosphate buffer, $\mathrm{pH}$ 7.5) were utilized by incubating for different time intervals at different temperatures.

\section{Lycopene production by wild-type IDI and its variant in batch fermentation}

A Biostat B plus MO5L fermenter (Sartorius Stedim Biotech GmbH, Göttingen, Germany) was used to perform fed-batch fermentation reactions using $2 \mathrm{~L}$ of fresh M9 medium (20 g glucose, $9.8 \mathrm{~g} \mathrm{KH}_{2} \mathrm{PO}_{4}, 2.1 \mathrm{~g}$ citric acid, $0.3 \mathrm{~g}$ ammonium ferric citrate, $5 \mathrm{~g}$ beef extract, $25 \mathrm{mg} \mathrm{MgSO}_{4} \cdot 7 \mathrm{H}_{2} \mathrm{O}$ per $1 \mathrm{~L}$ water, $\left.\mathrm{pH} 7.0\right)$ at $37{ }^{\circ} \mathrm{C}$. After the initial carbon source $(20 \mathrm{~g} / \mathrm{L}$ glucose $)$ was almost entirely consumed, a $3 \mathrm{M}$ glucose solution was added to begin the fed batch mode. A $25 \%$ ammonia solution was used to maintain a constant neutral $\mathrm{pH}$. Given that oxygen is an important factor for isoprenoid synthesis, fermentation was performed under strict aerobic conditions, with the dissolved oxygen concentration maintained at $20 \%$ saturation. When the $\mathrm{OD}_{600}$ reached 20, $0.05 \mathrm{mM}$ IPTG was added to induce recombinant protein expression. Fresh IPTG and antibiotics were added every $24 \mathrm{~h}$, and lycopene production and cell growth were monitored every $5 \mathrm{~h}$ throughout the $72 \mathrm{~h}$ fermentation process [35].

\section{Simulation of 3D structure and molecular docking of substrate of mutant IDI}

The NCBI protein database (http://www.ncbi.nlm.nih. gov/protein/) was utilized to search the amino acid sequence of IDI from S. cerevisiae. The BLAST server (http://blast.ncbi.nlm.nih.gov) was utilized to search a template for the chain. We applied E. coli IDI (PDB ID: 2ICJ) as the template [36], and the homology of amino acid sequence was determined by sequence alignment. Homology modeling of IDI was carried out using SWISS-MODEL.

An in silico molecular docking analysis was executed to investigate potential binding modes between IDI and IPP using Autodock vina 1.1.2. The tertiary and quaternary structure of IDI was built by SWISS-MODEL. The 2D structure of IPP was drawn by ChemBioDraw Ultra 14.0 and converted to 3D structure using ChemBio3D Ultra 14.0 software [37, 38]. Docking input files were created with the AutoDockTools 1.5.6 package [39]. The search grid for IPP was determined as center_x: 42.508, center_y: 33.926, and center_z: 6.015 with dimensions size_x: 15 , size_y: 15 , and size_z: 15 . The value of exhaustiveness was set to 20. Default Vina docking 
parameters were used unless otherwise described [40]. The best-scoring model as determined by the Vina docking score was visually analyzed using PyMoL 1.7.6 software (http://www.pymol.org/).

\section{Additional file}

Additional file 1. Methods, Tables and Figures.

\section{Abbreviations}

DMAPP: dimethylallyl pyrophosphate; GC: gas chromatography; IDI: isopentenyl diphosphate isomerase; IPP: isopentenylpyrophosphate; IPTG: isopropyl $\beta$-D-1-thiogalactopyranoside; MEP: 2-methyl-D-erythritol 4-phosphoric acid; MK: mevalonate kinase; MVA: mevalonate; MVD: diphosphomevalonate decarboxylase; PMK: phosphomevalonate kinase.

\section{Authors' contributions}

$\mathrm{HL}$ designed and operated the whole experiment, analyzed the data, and wrote the manuscript. MJ and CQ provided the experimental consideration and analyzed the partial data. MX and HB supervised the experiment process and reviewed the manuscript. $\mathrm{HZ}$ reviewed the manuscript. All authors read and approved the final manuscript.

\section{Author details \\ ${ }^{1}$ CAS Key Laboratory of Bio-based Materials, Qingdao Institute of Bioenergy and Bioprocess Technology, Chinese Academy of Sciences, No. 189 Songling Road, Qingdao 266101, People's Republic of China. ${ }^{2}$ Sino-Danish College, University of Chinese Academy of Sciences, No. 19(A) Yuquan Road, Bei- jing 100049, People's Republic of China.}

\section{Acknowledgements}

Not applicable.

\section{Competing interests}

The authors declare that they have no competing interests.

\section{Availability of data and materials}

The datasets supporting the conclusions of this article are included within the article.

\section{Consent for publication}

All the coauthors approved the submission/publication of this work to/in Microbial Cell factories.

\section{Ethics approval and consent to participate}

Not applicable.

\section{Funding}

This research was financially supported by National Natural Science Foundation of China (No. 21572242), Taishan Scholars Climbing Program of Shandong (No. tspd20150210), National Natural Science Foundation of China (NSF No. 31400084) and Youth Innovation Promotion Association CAS No. 2017252.

\section{Publisher's Note}

Springer Nature remains neutral with regard to jurisdictional claims in published maps and institutional affiliations.

Received: 20 December 2017 Accepted: 24 April 2018

Published online: 30 April 2018

\section{References}

1. Nowicka B, Kruk J. Occurrence, biosynthesis and function of isoprenoid quinones. Biochim Biophys Acta. 2010;1797:1587-605.
2. Bach TJ. Some new aspects of isoprenoid biosynthesis in plants. In: Kader JC, Mazliak P, editors. Plant lipid metabolism. Dordrecht: Springer; 1995. p. 321-34.

3. Rohmer M. The discovery of a mevalonate-independent pathway for isoprenoid biosynthesis in bacteria, algae and higher plants. Nat Prod Rep. 1999;16:565-74.

4. Kumar S, Hahn FM, Baidoo E, Kahlon TS, Wood DF, McMahan CM, Cornish K, Keasling JD, Daniell H, Whalen MC. Remodeling the isoprenoid pathway in tobacco by expressing the cytoplasmic mevalonate pathway in chloroplasts. Metab Eng. 2012;14:19-28.

5. Lichtenthaler HK, Schwender J, Disch A, Rohmer M. Biosynthesis of isoprenoids in higher plant chloroplasts proceeds via a mevalonateindependent pathway. FEBS Lett. 1997:400:271-4.

6. Berthelot K, Estevez Y, Deffieux A, Peruch F. Isopentenyl diphosphate isomerase: a checkpoint to isoprenoid biosynthesis. Biochimie. 2012:94:1621-34.

7. Rad SA, Zahiri HS, Noghabi KA, Rajaei S, Heidari R, Mojallali L. Type 2 IDI performs better than type 1 for improving lycopene production in metabolically engineered E. coli strains. World J Microbiol Biotechnol. 2012;28:313-21.

8. De Ruyck J, Wouters J, Poulter CD. Inhibition studies on enzymes involved in isoprenoid biosynthesis. Focus on two potential drug targets: $d x r$ and idi-2 enzymes. Curr Enzym Inhib. 2011;7:79-95.

9. Zhou C, Li Z, Wiberley-Bradford AE, Weise SE, Sharkey TD. Isopentenyl diphosphate and dimethylallyl diphosphate/isopentenyl diphosphate ratio measured with recombinant isopentenyl diphosphate isomerase and isoprene synthase. Anal Biochem. 2013;440:130-6.

10. Ramos-Valdivia AC, van der Heijden R, Verpoorte R. Isopentenyl diphosphate isomerase: a core enzyme in isoprenoid biosynthesis. A review of its biochemistry and function. Nat Prod Rep. 1997;14:591-603.

11. Zhao Y, Yang J, Qin B, Li Y, Sun Y, Su S, Xian M. Biosynthesis of isoprene in Escherichia coli via methylerythritol phosphate (MEP) pathway. Appl Microbiol Biotechnol. 2011;90:1915-22.

12. Ajikumar $P K$, Xiao WH, Tyo KE, Wang Y, Simeon F, Leonard E, Mucha O, Phon TH, Pfeifer B, Stephanopoulos G. Isoprenoid pathway optimization for Taxol precursor overproduction in Escherichia coli. Science. 2010;330:70-4

13. Yang J, Xian M, Su S, Zhao G, Nie Q, Jiang X, Zheng Y, Liu W. Enhancing production of bio-isoprene using hybrid MVA pathway and isoprene synthase in E. coli. PLoS ONE. 2012;7:e33509.

14. Yang J, Zhao G, Sun Y, Zheng Y, Jiang X, Liu W, Xian M. Bio-isoprene production using exogenous MVA pathway and isoprene synthase in Escherichia coli. Bioresour Technol. 2012;104:642-7.

15. Alper H, Jin YS, Moxley JF, Stephanopoulos G. Identifying gene targets for the metabolic engineering of lycopene biosynthesis in Escherichia coli. Metab Eng. 2005;7:155-64.

16. Bornscheuer UT, Pohl M. Improved biocatalysts by directed evolution and rational protein design. Curr Opin Chem Biol. 2001;5:137-43.

17. Hogrefe HH, Cline J, Youngblood GL, Allen RM. Creating randomized amino acid libraries with the QuikChange Multi Site-Directed Mutagenesis Kit. Biotechniques. 2002; 33:1158-60, 1162, 1164-5.

18. Anderson MS, Muehlbacher M, Street IP, Proffitt J, Poulter CD. Isopentenyl diphosphate:dimethylallyl diphosphate isomerase. An improved purification of the enzyme and isolation of the gene from Saccharomyces cerevisiae. J Biol Chem. 1989;264:19169-75.

19. Wu Z, Wouters J, Poulter CD. Isopentenyl diphosphate isomerase. Mechanism-based inhibition by diene analogues of isopentenyl diphosphate and dimethylallyl diphosphate. J Am Chem Soc. 2005;127:17433-8.

20. Street IP, Coffman HR, Baker JA, Poulter CD. Identification of Cys 139 and Glu207 as catalytically important groups in the active site of isopentenyl diphosphate:dimethylallyl diphosphate isomerase. Biochemistry. 1994;33:4212-7.

21. Lu XJ, Christensen DJ, Poulter CD. Isopentenyl-diphosphate isomerase: irreversible inhibition by 3-methyl-3,4-epoxybutyl diphosphate. Biochemistry. 1992;31:9955-60.

22. Janczak MW, Poulter CD. Kinetic and binding studies of streptococcus pneumoniae type 2 isopentenyl diphosphate: dimethylallyl diphosphate isomerase. Biochemistry. 2016;55:2260-8.

23. Kittleman W, Thibodeaux CJ, Liu YN, Zhang H, Liu HW. Characterization and mechanistic studies of type II isopentenyl diphosphate: dimethylallyl diphosphate isomerase from Staphylococcus aureus. Biochemistry. 2007:46:8401-13. 
24. Lv X, Xie W, Lu W, Guo F, Gu J, Yu H, Ye L. Enhanced isoprene biosynthesis in Saccharomyces cerevisiae by engineering of the native acetyl-COA and mevalonic acid pathways with a push-pull-restrain strategy. J Biotechnol. 2014;186:128-36.

25. Stemmer WPC. Rapid evolution of a protein in vitro by DNA shuffling. Nature. 1994;370:389-91.

26. Parnas $\mathrm{DL}$, Clements $\mathrm{PC}$. A rational design process: how and why to fake it. IEEE Trans Soft Eng. 1986;SE-12:251-7.

27. Reetz MT, Soni P, Fernández L, Gumulya Y, Carballeira JD. Increasing the stability of an enzyme toward hostile organic solvents by directed evolution based on iterative saturation mutagenesis using the B-FIT method. Chem Commun (Camb). 2010;46:8657-8.

28. Reetz MT, Carballeira JD. Iterative saturation mutagenesis (ISM) for rapid directed evolution of functional enzymes. Nat Protoc. 2007;2:891-930.

29. Chotani GK, Nielsen A, Sanford KJ. Reduction of carbon dioxide emission during isoprene production by fermentation. United States patent US 8470581. 2013. http://www.freepatentsonline.com/8470581.html. Accessed 19 Dec 2017.

30. Sadler G, Davis J, Dezman D. Rapid extraction of lycopene and $\beta$-carotene from reconstituted tomato paste and pink grapefruit homogenates. J Food Sci. 1990;55:1460-1.

31. Alper H, Miyaoku K, Stephanopoulos G. Characterization of lycopeneoverproducing $E$. coli strains in high cell density fermentations. Appl Microbiol Biotechnol. 2006;72:968-74.

32. de Ruyck J, Janczak MW, Neti SS, Rothman SC, Schubert HL, Cornish RM, Matagne A, Wouters J, Poulter CD. Determination of kinetics and the crystal structure of a novel type 2 isopentenyl diphosphate: dimethylallyl diphosphate isomerase from streptococcus pneumoniae. ChemBioChem. 2014;15:1452-8.

33. Cunningham FX, Sun Z, Chamovitz D, Hirschberg J, Gantt E. Molecular structure and enzymatic function of lycopene cyclase from the cyanobacterium Synechococcus sp. strain PCC7942. Plant Cell. 1994;6:1107-21.

34. Berthelot K, Estevez Y, Quiliano M, Baldera-Aguayo PA, Zimic M, Pribat A, Bakleh ME, Teyssier E, Gallusci P, Gardrat C, et al. HbIDI, SIIDI and EcIDI: a comparative study of isopentenyl diphosphate isomerase activity and structure. Biochimie. 2016;127:133-43.

35. Lacmata ST, Kuiate JR, Ding Y, Xian M, Liu H, Boudjeko T, Feng X, Zhao G. Enhanced poly (3-hydroxypropionate) production via $\beta$-alanine pathway in recombinant Escherichia coli. PLoS ONE. 2017;12:e0173150.

36. Voynova NE, Fu Z, Battaile KP, HerdendorfTJ, Kim JJ, Miziorko HM. Human mevalonate diphosphate decarboxylase: characterization, investigation of the mevalonate diphosphate binding site, and crystal structure. Arch Biochem Biophys. 2008;480:58-67.

37. Ibezim A, Nwodo NJ, Nnaji NJ, Ujam OT, Olubiyi OO, Mba CJ. In silico investigation of morpholines as novel class of trypanosomal triosephosphate isomerase inhibitors. Med Chem Res. 2017;26:180-9.

38. Morris GM, Huey R, Lindstrom W, Sanner MF, Belew RK, Goodsell DS, Olson AJ. AutoDock4 and AutoDockTools4: automated docking with selective receptor flexibility. J Comput Chem. 2009;30:2785-91.

39. Cleland WW. Statistical analysis of enzyme kinetic data. Methods Enzymol. 1979;63:103-38.

40. Sanner MF. Python: a programming language for software integration and development. J Mol Graph Model. 1999;17:57-61.
Ready to submit your research? Choose BMC and benefit from:

- fast, convenient online submission

- thorough peer review by experienced researchers in your field

- rapid publication on acceptance

- support for research data, including large and complex data types

- gold Open Access which fosters wider collaboration and increased citations

- maximum visibility for your research: over 100M website views per year

At BMC, research is always in progress.

Learn more biomedcentral.com/submissions 